\title{
Tourismus und Richtplanung am Beispiel des Berner Oberlandes
}

\section{Die Richtplanung und der Einbezug des Tourismus}

"Das oberste Ziel der Fremdenverkehrspolitik besteht in der langfristigen Gewährleistung der körperlichen und physischen Erholung sowie regenerierender Aktivität und Geselligkeit für möglichst breite Bevölkerungsschichten in intakter Landschaft und in sowohl Landschafts- wie Gästebedürfnissen angepaßten Siedlungsformen unter Berücksichtigung der langfristigen Interessen der ansässigen Bevölkerung» (KRIPPENDORF 1975).

Diese Zielsetzung gilt es im Rahmen der Richtplanung mit Zielsetzungen anderer Bereiche zu konfrontieren und bezüglich ihrer räumlichen Erfordernisse umzusetzen. Seit Inkrafttreten des Bundesgesetzes über die Raumplanung (RPG) vom 22. Juni 1979 gibt es für jeden geographischen Raum nur noch einen Richtplan, ob dieser nun schwergewichtig durch die Gemeinde, die Region oder den Kanton erarbeitet wird. Allerdings sind dabei so oder so die Interessen aller Ebenen zu berücksichtigen, d. h. beispielsweise sowohl die Grundsätze des schweizerischen Tourismuskonzeptes als auch die touristischen Leitbilder der einzelnen Kurorte. Dieser Richtplan «hält für raumwirksame Tätigkeiten das Ergebnis der kantonalen Planung und der Zusammenarbeit mit Bund und Nachbarkantonen fest. Er zeigt, wie Bund, Kantone und Gemeinden ihre raumwirksamen Zuständigkeiten - soweit sie einander berühren - wahrnehmen sollen, damit sie sich im Hinblick auf die erwünschte räumliche Entwicklung zu sinnvoller Ordnung fügen» (Erläuterungen zum RPG).

Der Einbezug der fremdenverkehrspolitischen Zielsetzung in die Richtplanung ist demzufolge soweit zwingend, als dadurch die räumliche Entwicklung eines Gebietes berührt werden kann. Dies ist insbesondere der Fall bei der Beurteilung der Belastbarkeit von Natur- und Siedlungsraum durch touristische Aktivitäten vor dem Hintergrund unterschiedlicher, zum Teil divergierender Bedürfnisse von Erholungssuchenden, der einheimischen Bevölkerung und der natürlichen Gegebenheiten selbst. Allerdings - und hier beginnen die Schwierigkeiten - sind handlungsorientierte Grundsätze und Konsequenzen für die Richtplanung in Fremdenverkehrsgebieten nicht unmittelbar aus Analysen touristischer Bedürfnisse und Verhaltensweisen abzuleiten: «Die verbindende Brücke zu konkreten und praxisbezogenen Regeln und Rezepten für planerische und gestalterische
Maßnahmen in Erholungsgebieten muß noch stets neu geschaffen werden. Bausteine dazu bilden - und darüber mag der reine Theoretiker zunächst enttäuscht sein - die Erfahrung und ein kritisches Verhältnis zur beobachteten touristischen Praxis» (BAUMGARTNER 1976).

\section{Die Region Oberland-Ost als Beispiel}

Die Berner Region Oberland-Ost umfaßt die Quellgebiete von Aare und Lütschine bis zum oberen Thunersee und ist ein Zusammenschluß von 28 Gemeinden (Fig. 1). Sie weist eine Fläche von rund $1300 \mathrm{~km}^{2}$ mit 41000 Einwohnern auf und zählt zu den bedeutendsten Fremdenverkehrsgebieten der Schweiz.

Jede Talschaft, ja sogar jeder der über 30 Fremdenorte, besitzt seinen eigenen Charakter und konnte seine Eigenart und sein Brauchtum über Jahrhunderte wahren. Somit präsentiert sich auf engstem Raum ein vielfältiges Feriengebiet mit unzähligen Erlebnisberei-

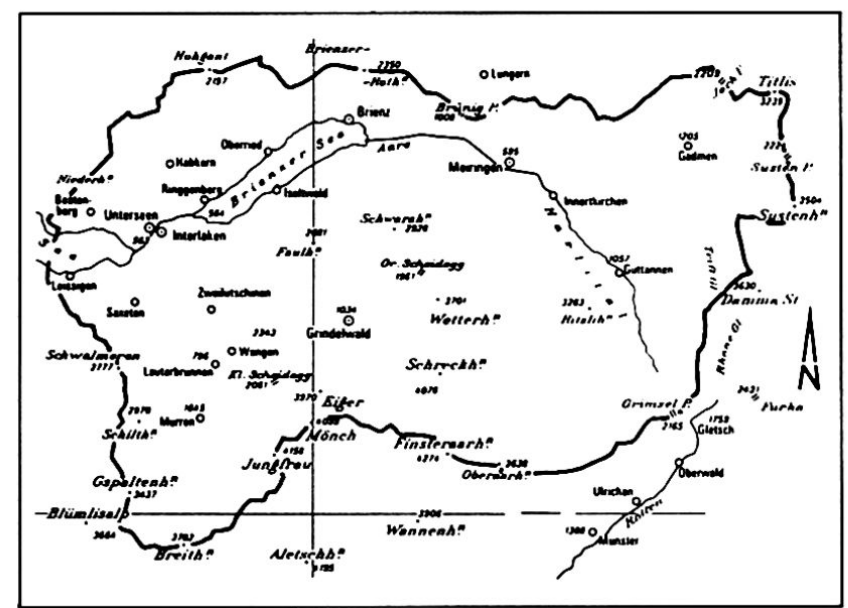

Fig. 1 Abgrenzung der Region Oberland-Ost

Ulrich Roth und Dr. Peter Hitz, Sigmaplan, AG für Raumplanung, Umweltplanung, Verkehrsplanung, Zähringerstr. 61, 3012 Bern 
chen. Davon zeugen auch die weit über 2 Mio. Logiernächte pro Jahr. Seit 1976 verfügt die Region über ein genehmigtes Entwicklungskonzept gemäß Bundesgesetz über Investitionshilfe für Berggebiete (IHG), mit dessen Hilfe bis heute ein Investitionsvolumen von rund 175 Mio. Franken ausgelöst werden konnte. Als raumplanerische Ergänzung zum schwergewichtig wirtschaftlich orientierten Entwicklungskonzept wurde bereits damals die Erarbeitung eines Richtplanes postuliert. Dieses Anliegen hat dann in Zusammenhang mit der Aufhebung des Bundesbeschlusses über dringliche Maßnahmen auf dem Gebiete der Raumplanung sowie mit dem Inkrafttreten des RPG an Aktualität gewonnen.

\subsection{Zweck der Richtplanung}

Die Richtplanung dient bei raumplanerischen Entscheiden als Koordinations- und Entscheidungsgrundlage. Damit soll ein ständiges und zielgerichtetes Gespräch zwischen den verschiedenen, mit raumwirksamen Aufgaben betrauten Stellen ermöglicht werden. Auf unsere Region bezogen, läßt sich dies stichwortartig wie folgt konkretisieren:

- räumliche Umsetzung der im Entwicklungskonzept formulierten Ziele

- Darstellung und Koordination der verschiedenartigen Interessen an räumlich begrenzten Flächen (z. B. Tourismus, Landwirtschaft, Baugebiet, Militär, Landschaftsschutz) und Aufzeigen möglicher Konfliktsituationen als Grundlage für deren Lösung

- Koordination der oft auf die Baugebiete beschränkten Ortsplanungen im Hinblick auf die regionale Interessenlage

aktive Mitwirkung der Region und der Gemeinden an dem gemäß RPG zu erarbeitenden kantonalen Richtplan. Im Kanton Bern ist die Erarbeitung des Richtplanes an die betreffenden Regionen delegiert worden. Dieses Vorgehen ermöglicht eine Planung von "unten nach oben», die Gemeinden können wichtige Inhalte der übergeordneten Planung maßgebend vorbestimmen.

Gerade für eine Bergregion scheint es uns wesentlich zu sein, im Richtplan nicht nur einen anzustrebenden, konfliktfreien Zielzustand zu sehen, sondern vielmehr ein Instrument, das erlaubt, Problemsituationen und die sich daraus ergebenden Konflikte so aufzuarbeiten und zu dokumentieren, daß für viele zukünftige Maßnahmen die notwendigen Entscheidungsgrundlagen zur Verfügung stehen.

\subsection{Randbedingungen für den Tourismus}

Der Tourismus ist auf eine Landschaft angewiesen, welche sich zur Ausübung der gewünschten Erholungstätigkeiten eignet. Dabei sind sowohl die Anforderungen des Winter- als auch des Sommertourismus zu berücksichtigen. Ein einsaisonaler Tourismus ist in den Berggebieten infolge der ungünstigen Auslastung des entsprechenden Angebots langfristig kaum tragbar. Es sind also einerseits vielfältige Wintersportmöglichkeiten mit den notwendigen Anlagen zu schaffen, anderseits die landschaftlichen Eindrücke, die Aussichtsmöglichkeiten, die gepflegten Wiesen, Weiden und Wälder, die saubere Luft, die Ruhe usw., kurz die naturnahe Landschaft als Gegensatz zum städtischen Lebensraum zu erhalten.

Den natürlichen Lebensgrundlagen kommt in unserer Region eine ganz besondere Bedeutung zu, weil sie sich als Ressource unmittelbarer in wirtschaftlichen Folgen äußert, als dies im Unterland im allgemeinen der Fall ist.

Die Erholungstätigkeiten sind der land- und forstwirtschaftlichen Nutzung im allgemeinen überlagert und haben deren spezifischen Belange gebührend $\mathrm{zu}$ berücksichtigen, um schwerwiegende Beeinträchtigungen zu verhindern. Das gilt ebenfalls für die verschiedenartigen touristischen Aktivitäten, die sich gegenseitig räumlich überlagern können.

Die nachhaltige Bewirtschaftung der Landschaft ist also einerseits das Resultat einer optimalen land- und forstwirtschaftlichen Nutzung, anderseits ist sie Voraussetzung für die touristischen Aktivitäten, welche zur wirtschaftlichen und infrastrukturellen Erhaltung und Förderung der Region unerläßlich sind.

Im Rahmen der Richtplanung muß daher generell umso mehr Gewicht auf Schutz und Pflege der Landschaft gelegt werden, je intensiver die touristische Entwicklung der Region ist. Im Gegensatz dazu finden wir - nicht nur bei der einheimischen Bevölkerung - die Idee noch weitverbreitet, daß die Prioritäten in einer Region entweder auf die touristische Entwicklung oder auf Schutz und Gestaltung der Landschaft zu legen seien. Nicht zuletzt deshalb hat sich in weiten Kreisen eine Angst vor vermeintlicher «Zukunftsverbauung» durch die Anliegen von seiten eines oft falsch verstandenen Landschaftsschutzes breitgemacht.

Die Funktion der Landschaft als Grundlage für eine solche Entwicklung wurde bereits erläutert. Zur Bereinigung der lokal auftretenden Konflikte zwischen den Bedürfnissen des Tourismus und der nachhaltigen Nutzung und Bewirtschaftung der Landschaft ist eine räumliche Entflechtung der widersprüchlichen Interessen innerhalb der Region anzustreben. Dies bedeutet, daß einerseits Gebiete zu bezeichnen sind, in welchen die natürliche Charakteristik der Landschaft möglichst unverändert erhalten bleiben soll. Anderseits sind gewisse hiefür speziell geeignete Gebiete einer intensiven touristischen Erschließung zugänglich zu machen. Dabei ist zu beachten, daß der extensive Tourismus, die ruhigen, nichttechnisierten, motorlosen Erholungsformen in letzter Zeit stark an Beliebtheit zunahmen. Die Forderung nach Erhaltung naturnaher Landschaftsgebiete ist also auch aus dieser Sicht zu unterstützen. 


\subsection{Vorgehen}

In Anbetracht der Vielzahl an Informationen, die in der als Gesamtplanung aufzufassenden Richtplanung zu verarbeiten sind, drängte sich eine den spezifischen Verhältnissen der Region Rechnung tragende Gliederung auf (Fig. 2).

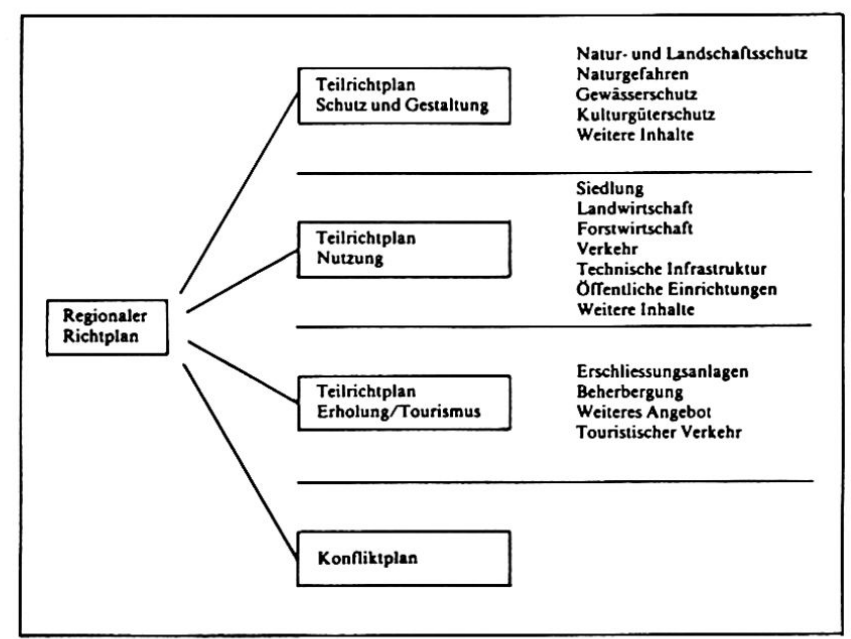

Fig. 2 Gliederung des Richtplaninhaltes

Im Bereich Tourismus wurden - wegen der erforderlichen gegenseitigen Abstimmung der Kapazitäten - die verschiedenen Aussagen bereits im Rahmen des Entwicklungskonzeptes quantifiziert. Aus diesen Angaben ließ sich für die Richtplanung eine Größenordnung der Nachfrage nach geeigneten Gebieten für die verschiedenen touristischen Aktivitäten ableiten. Dabei ging man von sämtlichen in Diskussion stehenden touristischen Projekten aus, welche einzeln durch einen aus den zuständigen Fachleuten der Region gebildeten «Fachausschuß Fremdenverkehr» beurteilt und den betroffenen Gemeinden zur Stellungnahme vorgelegt wurden.

Der Teilrichtplan «Schutz und Gestaltung» hatte aufzuzeigen, inwiefern und in welchen Gebieten diese Nachfrage überhaupt befriedigt werden kann und soll. In unserer Region drängte sich in erster Linie eine Abgrenzung zwischen den für den alpinen Skisport benötigten Gebieten und den aus landschaftlicher Sicht nicht mit touristischen Anlagen zu erschließenden Räumen auf. Für die Erholungstätigkeiten im Sommer sind im allgemeinen nicht derart schwerwiegende Eingriffe in die Landschaft notwendig wie für den alpinen Skisport (mechanische Erschließungsanlagen, Skipistenplanierungen usw.). Vom Sommertouristen werden im Gegenteil immer mehr naturnahe, unverbaute Landschaften - die auch durch Einrichtungen für den Wintersport nicht beeinträchtigt sein sollten - bevorzugt. Bereits im Entwicklungskonzept waren deshalb die folgenden Gebietskategorien räumlich grob festgelegt worden, welche im Rahmen der Richtplanung zu konkretisieren waren:

- Erholungsräume mit bedeutenden touristischen Anlagen: Gebiete, in denen die im Interesse des Tourismus unerläßlichen Eingriffe (Transportanlagen, Skipistenplanierungen, größere Gaststätten) unter weitgehender Schonung der Landschaft vorgenommen werden können. Im allgemeinen handelt es sich hierbei um Ergänzungen von touristisch bereits erschlossenen Gebieten. Die Mechanisierung von touristisch noch unerschlossenen Gebieten wird auch von seiten der touristischen Fachleute als unerwünscht und wirtschaftlich unzweckmäßig erachtet.

- Landschaftsschongebiete und Erholungsräume ohne bedeutende touristische Anlagen: Attraktive Landschaften, die im allgemeinen landwirtschaftlich genutzt werden und auch für die Erholung geeignet sind. Die für die extensiven Tourismusformen erforderlichen Anlagen (Wanderwege, Parkplätze an Ausgangspunkten, Feuerstellen, kleinere Spielund Lagerplätze usw.) sind den landschaftlichen Zielsetzungen unterzuordnen.

- Natur- und Landschaftsschutzgebiete: Gebiete von besonderer ökologischer, ästhetischer, natur- und kulturhistorischer Bedeutung, in denen keine der Schutzfunktion des Gebietes widersprechende Anlagen erstellt werden sollen.

- Schutz des Hochgebirges: Das Hochgebirge stellt von wenigen Ausnahmen abgesehen - die letzte großräumig zusammenhängende und weitgehend unberührte Naturlandschaft unseres Landes dar. Die Eingriffe durch technische Bauwerke und touristische Erschließungen sind hier auch bei standortgebundenen Anlagen (z. B. Restaurantneubau auf dem Jungfraujoch) auf ein Minimum zu beschränken.

Bei der Bereinigung von Widersprüchen zwischen landschaftlichen und touristischen Zielsetzungen sind die Interessen aller Beteiligten (vor allem auch von seiten der Land- und Fortwirtschaft) zu koordinieren. Dem gegenseitigen Verständnis kommt dabei sehr große Bedeutung zu. Hier kommen vor allem die politischen Interessen zum Zuge, welche für die spätere Realisierung der formulierten Ziele bereits in diesem Stadium von eminenter Bedeutung sind.

Wesentlich erscheint uns insbesondere, da $\beta$ bei allen Beteiligten Klarheit herrscht über die Bedeutung bezeichneter Gebiete, die auch langfristig nicht durch touristische Bauten und Anlagen verändert werden sollen. Dazu muß - speziell in einer auf den Tourismus ausgerichteten Region - die Erkenntnis wachsen, daß gerade solche Oasen für den Tourismus selbst je länger, je mehr von Bedeutung sind. 
Durch die Bezeichnung derartiger Gebiete treten neue, bisher noch nicht gelöste Probleme auf. Auch zwischen Fremdenverkehrsgemeinden und Gemeinden ohne touristische Infrastrukturanlagen besteht ein Wohlstandsgefälle. Erhaltenswerte und noch weitgehend unberührte Landschaften finden wir vorwiegend in touristisch weniger entwickelten Gemeinden. Damit entsteht die Gefahr der Festigung, ja sogar der Verstärkung eines innerregionalen Entwicklungsgefälles. Größere Investitionen wirtschaftlicher und damit auch touristischer Art sind in den landschaftlich reizvollen Gebieten ja eben gerade nicht erwünscht. Soll die Erhaltung und Pflege naturnaher Landschaftsräume nachhaltig gewährleistet werden, so ist ein verstärkter Finanzausgleich anzustreben, der die erwähnten innerregionalen Differenzen mindert.

\section{Schlußfolgerungen}

Aufgrund der vorstehenden Ausführungen wie auch der gesammelten Erfahrungen in diesem Bereich lassen sich die wesentlichen Anliegen anhand der folgenden Grundsätze zusammenfassen (vgl. hiezu auch BAUMGARTNER 1976):

- Ziele und Grundsätze der touristischen Planung müssen in einem wechselseitigen Abhängigkeitsverhältnis zu den Zielen und Grundsätzen anderer Șachplanungen (wie Landwirtschaft, Verkehr usw.) und den übergeordneten Zielen und Grundsätzen der Raumordnung (vgl. z. B. Art. 1 und 3 RPG) stehen. Konflikte sind als solche deutlich darzustellen.

- Ziele und Grundsätze der touristischen Planung sowie deren Berücksichtigung in der Richtplanung bedürfen der ständigen Anpassung an neue Erkenntnisse (wie Belastungsgrenzwerte) und an neue bzw. veränderte Bedürfnisse von Einheimischen und Erholungssuchenden.
- Ziel der touristischen Planung muß es sein, einen Beitrag zur optimalen Befriedigung der drei touristischen Hauptbedürfnisse - Erholungs- und Ruhebedürfnis, Bedürfnis nach Abwechslung und Ausgleich, Bedürfnis nach Befreiung von den $Z$ wängen des Alltages - zu leisten.

- Die touristische Planung sowie deren Berücksichtigung in der Richtplanung setzen eine aktive Mitwirkung der einheimischen Bevölkerung voraus.

- Wohn- und Arbeitsplätze der einheimischen Bevölkerung sollen im touristischen Siedlungsgebiet integriert sein, eine touristische Ghettobildung ist zu vermeiden.

- Das Flächenwachstum des Siedlungsgebietes soll begrenzt werden. Kriterien hiezu bilden nicht nur die Belastungsgrenzen der Erholungslandschaft und die Bedürfnisse der Einheimischen im Hinblick auf die Dominanz des Tourismus, sondern auch die «Orientierungskapazität» des für kurze Zeit anwesenden Touristen.

- Das Siedlungsgebiet wie auch die Erholungslandschaft müssen den Bedürfnissen nach Ruhe und Erholung durch optimale Immissionsfreiheit entsprechen können. (Berücksichtigung bei der Lenkung des rollenden und ruhenden Verkehrs, bei der Ausbreitung des Heliskiings, bei militärischen Schießübungen usw.)

Diese Grundsätze sind im Richtplan je nach Gebiet und politischem Willen mit mehr oder weniger Gewicht zu beachten. Nur so läßt sich eine optimale Berücksichtigung aller Faktoren erreichen, die zu einer wünschbaren Entwicklung eines Raumes sowohl für die ansässige Bevölkerung wie,auch hinsichtlich Landschafts- und Gästebedürfnissen beitragen können.

\section{Literatur}

BAUMGARTNER F. und KRIPPENDORF J. (1976): Bedürfnisse und Verhaltensweisen der touristischen Nachfrage und ihre Konsequenzen für die Raumplanung, Schweiz. Fremdenverkehrsverband, Dokumentations- und Beratungsstelle, Bern.

BUNDESAMT FÜR RAUMPLANUNG (1981): Erläuterungen zum Bundesgesetz über die Raumplanung, Eidg. Drucksachen- und Materialzentrale, Bern.
KRIPPENDORF J. (1975): Die Landschaftsfresser, Tourismus und Erholungslandschaft - Verderben oder Segen? Verlag Hallwag, Bern und Stuttgart.

ROTH U. (1981): Richtplanung im Berggebiet - Beispiel Region Berner Oberland-Ost. In: Schweizer Ingenieur und Architekt, Heft 10. 